\title{
Ex vivo micro-computed tomography analysis of bleomycin-induced lung fibrosis for preclinical drug evaluation
}

\author{
Chris J. Scotton ${ }^{1}$, Brian Hayes², Robert Alexander ${ }^{1}$, Arnab Datta', Ellen J. Forty ${ }^{1}$, \\ Paul F. Mercer ${ }^{1}$, Andy Blanchard ${ }^{2}$ and Rachel C. Chambers ${ }^{1}$
}

Affiliations: ${ }^{1}$ Centre for Inflammation and Tissue Repair, UCL Respiratory, University College London, London, and ${ }^{2}$ Fibrosis DPU, GlaxoSmithKline, Stevenage, UK.

Correspondence: C.J. Scotton, Centre for Inflammation and Tissue Repair, UCL Respiratory, University College London, 5 University Street, London, UK. E-mail: c.scottonQucl.ac.uk

ABSTRACT Research into the pathogenesis underlying the development of idiopathic pulmonary fibrosis is hampered by a repertoire of animal models that fail to recapitulate all the features of the human disease. Better use and understanding of what the animal models represent may improve clinical predictability. We interrogated ex vivo micro-computed tomography $(\mathrm{CT})$ as a novel end-point measure in the mouse model of bleomycin-induced lung fibrosis (BILF), and to evaluate a therapeutic dosing regimen for preclinical drug evaluation.

A detailed characterisation of BILF was performed using standard end-point measures (lung hydroxyproline and histology). High resolution micro-CT $(\sim 13.7 \mu \mathrm{m}$ voxel size $)$ was evaluated for quantifying the extent and severity of lung fibrosis.

The period from 14 to 28 days following bleomycin instillation represents progression of established fibrosis. A therapeutic dosing regimen during this period was validated using a transforming growth factor- $\beta$ receptor-1 kinase inhibitor, and micro-CT provided a highly sensitive and quantitative measure of fibrosis. Moreover, fibrotic lesions did not completely resolve, but instead persisted for $\geqslant 6$ months following a single insult with bleomycin.

Ex vivo micro-CT analysis of BILF allows robust evaluation of therapeutic dosing once fibrosis is already well established, requiring fewer mice than conventional biochemical end-points.

@ERSpublications

Micro-CT has improved out understanding of the bleomycin model of fibrotic lung disease for preclinical drug evaluation http://ow.ly/p73De

This article has supplementary material available from www.erj.ersjournals.com

Received: Nov 122012 | Accepted after revision: Feb 042013 | First published online: March 212013

Support statement: The authors are grateful to the following grant agencies for funding: British Lung Foundation (C.J. Scotton Research Fellowship F07/6); Medical Research Council (C.J. Scotton Career Development Award G0800340; R.C. Chambers CASE Award 2009-2012); Wellcome Trust (A. Datta Clinical Research Training Fellowship 084382/Z/07/Z); European Commission (R.C. Chambers Framework 7 Programme HEALTH-F2- 2007-2224); and the European IPF Network). SB525334A was donated by GlaxoSmithKline (Stevenage, UK).

Conflict of interest: Disclosures can be found alongside the online version of this article at www.erj.ersjournals.com

Copyright OERS 2013. ERJ Open articles are open access and distributed under the terms of the Creative Commons Attribution Non-Commercial Licence 3.0. 


\section{Introduction}

Idiopathic pulmonary fibrosis (IPF) is a chronic, progressive and ultimately fatal condition, characterised by the excessive deposition of extracellular matrix and destruction of the lung architecture. In the USA and Europe there are currently $>500000$ IPF patients [1], who place a significant clinical and financial burden on healthcare systems. Disease progression is generally refractory to current pharmacological interventions, resulting in a median survival of 3-5 years following diagnosis [2]. Identification of novel therapeutic interventions is therefore paramount to supplement the extremely limited repertoire of drugs that have shown signs of clinical benefit.

Experimental lung fibrosis models are routinely used for the identification of relevant underlying pathomechanisms and assessment of drug efficacy. However, IPF research is hampered by animal models that do not recapitulate all the features of the human disease, and whose ability to predict future clinical outcomes has been questioned. From the plethora of pathways/targets that show antifibrotic potential in these experimental models, few have gone on to show benefit in clinical trials (see [3] for an excellent review). Several different models exist [4], of which lung fibrosis induced by bleomycin application is probably the best characterised and most widely utilised, although there is no accepted standardised version of this model [5]. A variety of factors will affect the nature of the fibrotic response, including the route of administration, the bleomycin preparation, dose, mouse strain, age and so on. Prophylactic dosing schedules and nonconditional genetically deficient mouse studies often report beneficial effects, but potentially not through a direct antifibrotic response, but instead by dampening the early inflammatory reaction to bleomycin. More therapeutically targeted dosing regimens are infrequently employed (e.g. pirfenidone [6], BIBF 1000 [7], imatinib [8] and prednisolone [9]), yet these have arguably provided a better prediction of clinical outcome.

Given the limited signal window of traditional fibrosis end-points, such as high-performance liquid chromatography (HPLC) determination of lung hydroxyproline, we employed micro-computed tomography (micro-CT) analysis of murine lungs ex vivo and established a quantitative end-point measurement of fibrotic change following oropharyngeal bleomycin administration. Moreover, an appropriate window for applying a therapeutic dosing regimen to block fibrotic progression was determined, and successfully benchmarked using a small molecule inhibitor of transforming growth factor$\beta$ receptor (TGF- $\beta R$ )-1/activin receptor-like kinase (ALK) 5 to block the signalling pathway downstream of the major pro-fibrotic cytokine, TGF- $\beta$. Using micro-CT, we also established that the fibrotic lesions did not completely resolve, but instead persisted for several months following a single insult with bleomycin.

\section{Materials and methods}

Detailed methods are provided in the online supplementary material.

\section{Bleomycin animal model of lung injury}

All animal studies were ethically reviewed and carried out in accordance with Animals (Scientific Procedures) Act 1986 and the GlaxoSmithKline (GSK) Policy on the Care, Welfare and Treatment of Animals. Bleomycin (50 IU $\cdot$ mouse $^{-1}$ in $50 \mu \mathrm{L}$ sterile $0.9 \%$ saline (Bleo-kyowa; Kyowa Hakko Ltd, Slough, $\mathrm{UK}$ ) or saline was administered by oropharyngeal instillation to male $\mathrm{C} 57 \mathrm{Bl} / 6$ mice (Charles River Laboratories, Margate, UK) as described previously [10]. The experimental groups are outlined in table 1.

For real-time reverse transcriptase (RT)-PCR and total lung collagen measurements, lungs were snap-frozen in liquid nitrogen and weighed. For micro-CT, histological and immunohistochemical analysis, lungs were insufflated and fixed, as described previously [10], although alternative fixation approaches exist for microCT imaging, e.g. [11].

\section{ALK5 inhibition studies using SB525334A}

The highly selective small molecule ALK5 inhibitor, SB525334A [12], was a gift from GSK (Stevenage, UK). 14 days post-bleomycin, SB525334A $\left(30 \mathrm{mg} \cdot \mathrm{kg}^{-1}\right.$ in $100 \mu \mathrm{L}$ acidified saline $/ 0.2 \%$ Tween $80 \mathrm{pH} \mathrm{4.1)}$ or vehicle (acidified saline/0.2\% Tween $80 \mathrm{pH} 4.1$ ) was administered twice daily by oral gavage, until 28 days post-bleomycin (see table 1 for experimental groups).

\section{Determination of total lung collagen}

Total lung collagen was determined by measuring hydroxyproline content in aliquots of pulverised lung as described previously [10]. Hydroxyproline was quantified by reverse-phase HPLC of 7-chloro-4nitrobenzo-oxao-1,3-diazole-derived acid hydrolysates. Total lung collagen was calculated assuming that collagen contains $12.2 \%(\mathrm{w} / \mathrm{w})$ hydroxyproline and was expressed as $\mathrm{mg}^{\circ} \mathrm{lung}^{-1}$. 
TABLE 1 Details of in vivo experimental groups, time points, mouse numbers and end-point analyses

\section{Experiment}

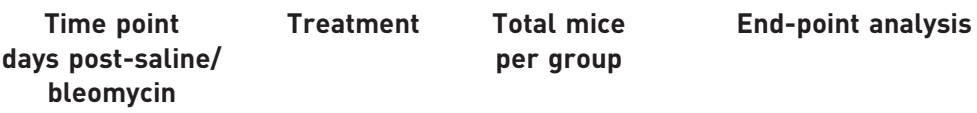

Timecourse of fibrotic response to bleomycin injury

Determination of resolution post-bleomycin injury

Investigation of therapeutic effects of ALK5 inhibitor (dosed from day 14 to day 28 post-bleomycin)

\begin{tabular}{|c|c|}
\hline 3 & Saline \\
\hline 3 & Bleomycin \\
\hline 7 & Saline \\
\hline 7 & Bleomycin \\
\hline 10 & Saline \\
\hline 10 & Bleomycin \\
\hline 4 & Saline \\
\hline 4 & Bleomycin \\
\hline 21 & Saline \\
\hline 21 & Bleomycin \\
\hline 28 & Saline \\
\hline 28 & Bleomycin \\
\hline 4 & Saline \\
\hline 4 & Bleomycin \\
\hline 68 & Saline \\
\hline 68 & Bleomycin \\
\hline 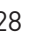 & Saline + vehicle \\
\hline 28 & $\begin{array}{c}\text { Saline + } \\
\text { SB525334A }\end{array}$ \\
\hline 28 & $\begin{array}{l}\text { Bleomycin + } \\
\text { vehicle }\end{array}$ \\
\hline 28 & $\begin{array}{l}\text { Bleomycin + } \\
\text { SB525334A }\end{array}$ \\
\hline
\end{tabular}

\author{
Biochemistry 10 , histology 3 \\ Biochemistry 11, histology 3 \\ Biochemistry 10 , histology 3 \\ Biochemistry 11, histology 3 \\ Biochemistry 8 , histology 3 \\ Biochemistry 9, histology 3 \\ Biochemistry 10, histology/ \\ micro-CT 4 \\ Biochemistry 8 , histology/ \\ micro-CT 5 \\ Biochemistry 10 , histology/ \\ micro-CT 4 \\ Biochemistry 8 , histology/ \\ micro-CT 5 \\ Biochemistry 10, histology/ \\ micro-CT 5 \\ Biochemistry 9, histology/ \\ micro-CT 5 \\ Biochemistry 8 , histology/ \\ micro-CT 7 \\ Biochemistry 8 , histology/ \\ micro-CT 7 \\ Histology/micro-CT 4 \\ Histology/micro-CT 4 \\ Biochemistry 5, histology/ \\ micro-CT 3 \\ Biochemistry 5, histology/ \\ micro-CT 3 \\ Biochemistry 8 , histology/ \\ micro-CT 4 \\ Biochemistry 7, histology/ \\ micro-CT 4
}

Data are presented as $\mathrm{n}$, unless otherwise stated. ALK: activin receptor-like kinase; micro-CT: micro-computed tomography.

Total lung collagen was also measured using the Sircol assay (Biocolor Ltd, Carrickfergus, UK) according to the manufacturer's instructions, and expressed as $\mathrm{mg} \cdot \mathrm{lung}^{-1}$.

\section{Micro-CT scanning}

Fixed, insufflated lungs were incubated for $2 \mathrm{~h}$ each in 70, 80 and 90\% ethanol and then overnight in 100\% ethanol. Lungs were subsequently transferred to $100 \%$ hexamethyldisilazane for a further $2 \mathrm{~h}$, prior to airdrying. Lungs were then packaged for micro-CT scanning. Voxel sizes of 1-2 $\mu \mathrm{m}$ are feasible [11], but associated with prohibitively long scan/reconstruction times of several hours per sample. We opted for a voxel size of $13.7 \mu \mathrm{m}$ and a scan time of $\sim 10 \mathrm{~min}$, allowing high-resolution visualisation and analysis of fibrosis, with good throughput. Lungs were scanned in a SkyScan 1072 micro-CT scanner (Bruker MicroCT, Kontich, Belgium) at $40 \mathrm{kV} \cdot 100 \mu \mathrm{A}^{-1}$, without a filter, using two-frame averaging and $0.9^{\circ}$ angular rotation step size; based on the typical measured $10 \%$ modulation transfer function in the SkyScan 1072, spatial resolution was in the region of 20-30 $\mu \mathrm{m}$. Reconstruction was carried out with the SkyScan NRecon software (Bruker MicroCT).

\section{Micro-CT image analysis}

Tissue segmentation analysis was performed using InForm software (PerkinElmer, Cambridge, UK). Briefly, the software was "trained" to recognise fibrotic or normal lungs, large airways, vessels and packaging material, using two representative micro-CT sections (8-bit greyscale) from each animal in the study. Segmentation was then carried out on a medium sample area at fine resolution, to measure the tissue 
volume and greyscale density of fibrotic and normal lung tissue for every micro-CT section. Data for each lung ( 900 sections) were then compiled into a composite measurement of fibrosis (volume fibrosis $\times$ greyscale density) per whole lung.

\section{Voxel density analysis}

Composite 256-colour greyscale histograms (from $0=$ black to $255=$ white) were generated for each lung (excluding the large airways), representing the frequency distribution of pixel densities. The mean number of pixels in each bin (1 greyscale unit wide) were then calculated for each group, along with a t-test distribution showing the p-value for the comparison between each animal group at each greyscale bin. Greyscale density was not converted to Hounsfield Units due to the processing and dehydration required for our ex vivo analysis.

\section{Histological analysis}

For standard histological processing (haematoxylin and eosin staining or Martius Scarlet Blue stain for collagen), 2- $\mu \mathrm{m}$ paraffin sections were cut and mounted on polylysine-coated glass slides and processed accordingly. All sections were subsequently scanned on a Nanozoomer and images were captured using NDP.view v. 1.2.36 (both from Hamamatsu Corporation, Hamamatsu, Japan). Direct comparisons between micro-CT and histology were performed on the same set of lungs; post-CT, lungs were rehydrated through an ethanol gradient $(100,90,80$ and $70 \% ; 2 \mathrm{~h}$ in each) prior to normal histological processing.

\section{Real-time RT-PCR}

Real-time quantitative (q)PCR was performed as previously described [10], except that normalisation used the geometric mean of the crossing point $(\mathrm{Cp})$ values for two housekeeping genes: ATP synthase $5 \mathrm{~B}$ and $\beta 2$ microglobulin, identified by GeNorm analysis as the most stable housekeeping genes for this study. Statistics were calculated on the $\Delta \mathrm{Cp}$ values. All primers and GeNorm kits were purchased from Primer Design (Southampton, UK).

\section{Statistical analysis}

Statistical analysis was performed using SigmaPlot 12.3 software (Systat Software, Inc., London, UK). Differences between two groups were evaluated by a two-tailed t-test. Multiple group comparisons were evaluated using one- or two-way ANOVA as appropriate, with Holm-Sidak post hoc testing. Pearson's correlation was used to determine the statistical significance of correlated data. A p-value $<0.05$ was considered significant.

\section{Results}

Delineation of the progressive fibrotic phase following bleomycin instillation

Our standard model of bleomycin-induced lung fibrosis (BILF) had typical end-points for the inflammatory and fibrotic phases of 7 and 14 days, respectively (associated with a $\sim 60 \%$ increase in the amount of total lung collagen). We chose to assess changes in lung parameters over an extended timecourse, from 3 days to 28 days post-bleomycin. In a separate experiment, we also assessed mice 3 months and 6 months after injury.

Total lung weight rose rapidly post-bleomycin, peaking at 14 days (fig. 1a). At 3 months post-bleomycin, lung weight was still significantly increased above control (mean \pm SEM of bleomycin versus saline $194 \pm 3.4 \mathrm{mg}$ versus $145 \pm 3.9 \mathrm{mg}, \mathrm{n}=8 ; \mathrm{p}<0.05)$. HPLC determination of hydroxyproline content demonstrated that total lung collagen was significantly increased above baseline from day 10 onwards (fig. 1b), with a $65 \%$ increase in collagen at day 14, rising to a $91 \%$ increase in total lung collagen by day 28 . In contrast, the commonly used Sircol assay gave a markedly different profile and magnitude of the changes in lung collagen (online supplementary fig. S1c), accounting for just $9 \%$ of the lung collagen determined by HPLC at day 28; no correlation was observed between HPLC and Sircol measures of lung collagen. Representative histological sections are depicted in online supplementary figure S2.

The period from day 14 to day 28, therefore, represents progression of established fibrosis, and was chosen as the optimal window of opportunity for therapeutic intervention in this model. Interestingly, total lung collagen remained significantly elevated at 3 months post-bleomycin, to the same extent (i.e. $\sim 92 \%$ increase over baseline) as that seen at day 28 , indicating a failure to reverse the increased deposition of lung collagen up to 12 weeks following a single bleomycin instillation.

\section{Ex vivo micro-CT analysis of the temporal response to bleomycin}

We sought to establish whether micro-CT could provide a more sensitive and reliable end-point measure of fibrotic change for the entire lung ex vivo, compared with HPLC analysis of hydroxyproline or histologic analysis. Representative mid-lung transverse micro-CT sections and 3D volume renderings from day 14 to 

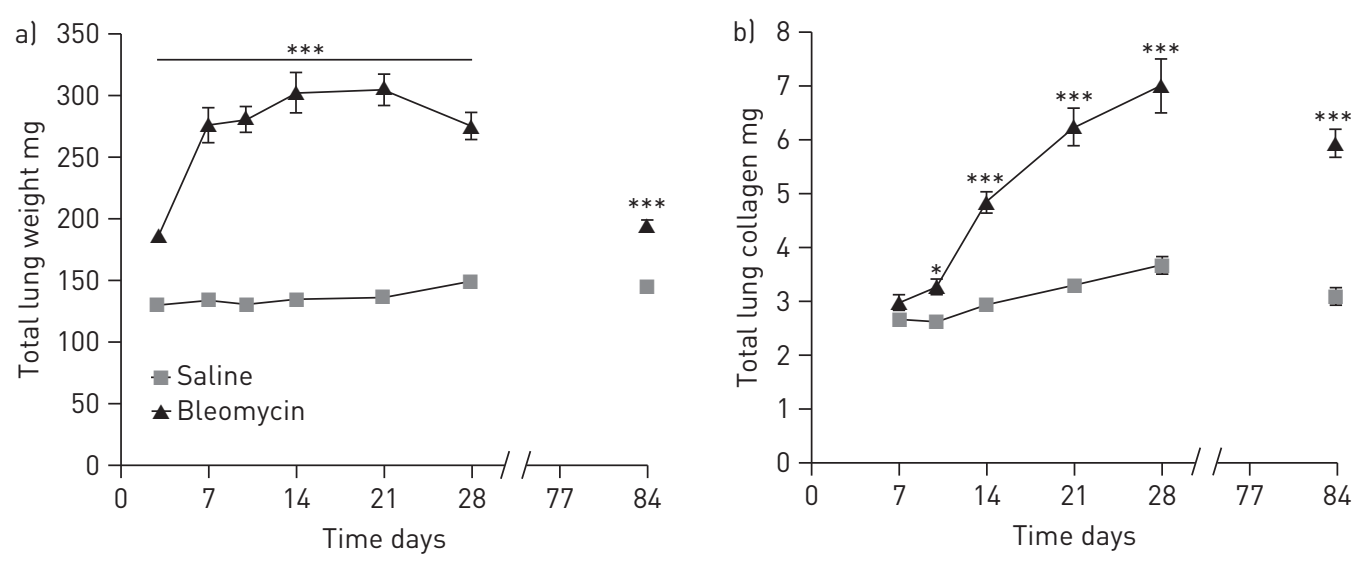

FIGURE 1 Timecourse of increase in lung weight and total lung collagen following bleomycin instillation. a) Lung weight increased rapidly after bleomycin injury, with a significant increase at day 3 post-injury, attributable to vascular leak and lung oedema, which was further augmented by day 7 and then maintained at a relatively stable level until day 28 , despite a concomitant decline in the degree of vascular leak in this model [10]. b) Total lung collagen (high-performance liquid chromatography determination of hydroxyproline) was significantly increased from day 10 to day 28. In a separate cohort of mice, the lung collagen content at 3 months post-bleomycin showed no diminution from the corresponding value at 28 days. There was a significant correlation between lung weight and total lung collagen at day $28\left(r^{2}=0.69 ; p<0.01\right)$. All data are presented as mean \pm SEM. $n=8-11$ mice per group. ${ }^{*}: p<0.05 ;{ }^{* * *}: \mathrm{p}<0.001$ (ANOVA versus matched time point control).

day 28 post-bleomycin are shown in figure 2. X-ray dense fibrotic lesions were clearly apparent, with a more apical and dorsocentric distribution attributable to the route of bleomycin distribution (fig. $2 \mathrm{~b}$ and online supplementary videos 1-4). Fibrosis was prominent around bronchovascular bundles and extended out to the periphery; evidence of traction bronchiectasis, dense consolidation, interlobular septal thickening and subpleural scarring were also easily discernible.

\section{ALK5 inhibition during the progressive phase of fibrosis}

As a proof of concept for quantifying the degree of fibrosis evident on micro-CT after an appropriately timed therapeutic intervention, a highly potent and selective small molecule inhibitor of TGF- $\beta$ R-1 (ALK5), SB525334A, was given twice daily by oral gavage from day 14, once fibrosis was already well-established, until day 28.

Bleomycin injury resulted in increased lung weight at day 28, but no significant differences were seen between the vehicle and ALK5 inhibitor groups (fig. 3a). However, therapeutic ALK5 inhibition significantly attenuated the BILF at day 28 , as evidenced by HPLC determination of lung hydroxyproline (fig. 3b). SB525334A significantly reduced (by 55\%), but did not fully reverse, the increased deposition of lung collagen.

Micro-CT detected extensive fibrotic change, which fully matched the equivalent histological sections (fig. 3e and f). We utilised a pattern recognition approach with InForm software to segment the tissue into normal parenchyma and fibrotic lesions (excluding extraneous fascia or packaging material), for all $\sim 14000$ micro-CT sections from the study (fig. 3d). We calculated the volume of lung occupied by fibrotic lesion, and its associated greyscale CT density, to create a composite measure of volume $\times$ density, encompassing both the extent and severity of fibrosis (fig. 3c). Micro-CT gave a much greater window for detecting fibrotic change compared with lung hydroxyproline, but the therapeutic effects of ALK5 inhibition were equivalent ( $\sim 60 \%$ reduction in fibrosis compared with bleomycin alone).

We also performed a simple voxel density analysis of each lung, without relying on segmentation into normal and fibrotic regions. Histograms were generated showing the mean number of voxels per lung, at each greyscale value from 0 (least dense) to 255 (most dense) (fig. $4 \mathrm{a}$ and b). The bleomycin + vehicle group had an increased proportion of higher greyscale values (indicative of higher density on CT) compared with the saline + vehicle group. In comparison with the bleomycin + vehicle group, the bleomycin + SB525334A mice clearly had significantly fewer voxels in the density range 42-137, indicating a lesser degree of fibrosis (fig. $4 \mathrm{~b}$ and c). No significant differences were detected between saline + vehicle and the saline + SB525334A groups (not shown).

\section{Micro-CT quantification of lungs 3 and 6 months post-bleomycin}

We also used micro-CT to address the question of resolution and restoration of normal lung architecture following a single instillation of bleomycin. At both 3 and 6 months post-bleomycin, the persistence of 
a)
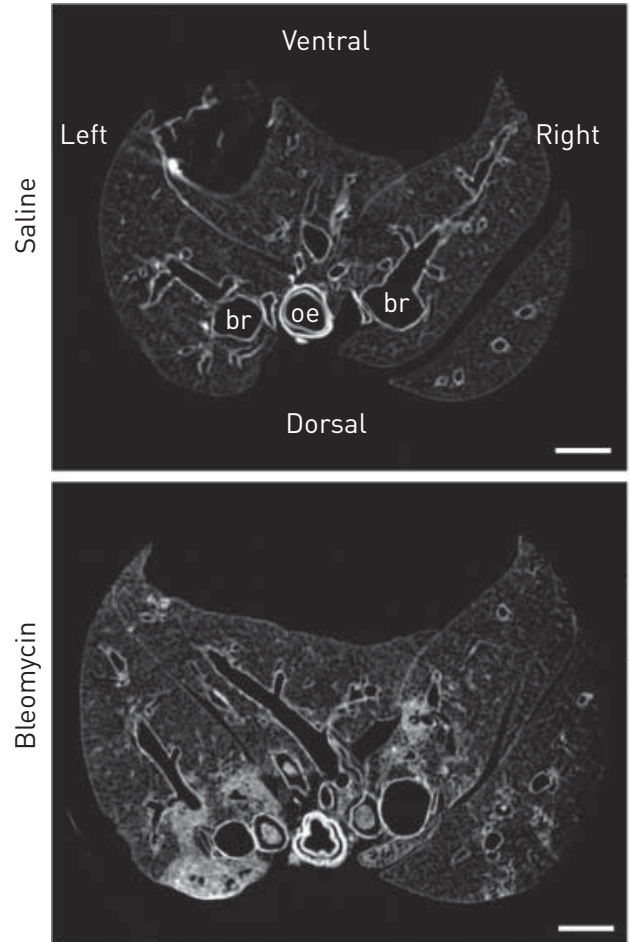

b)

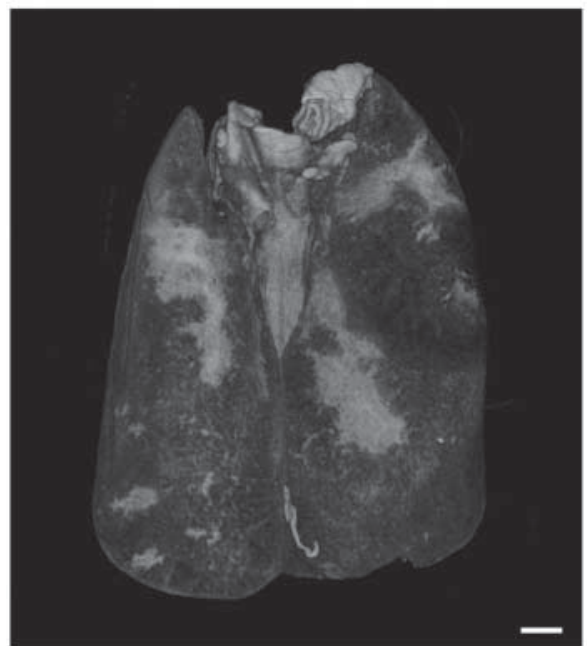

Day 21
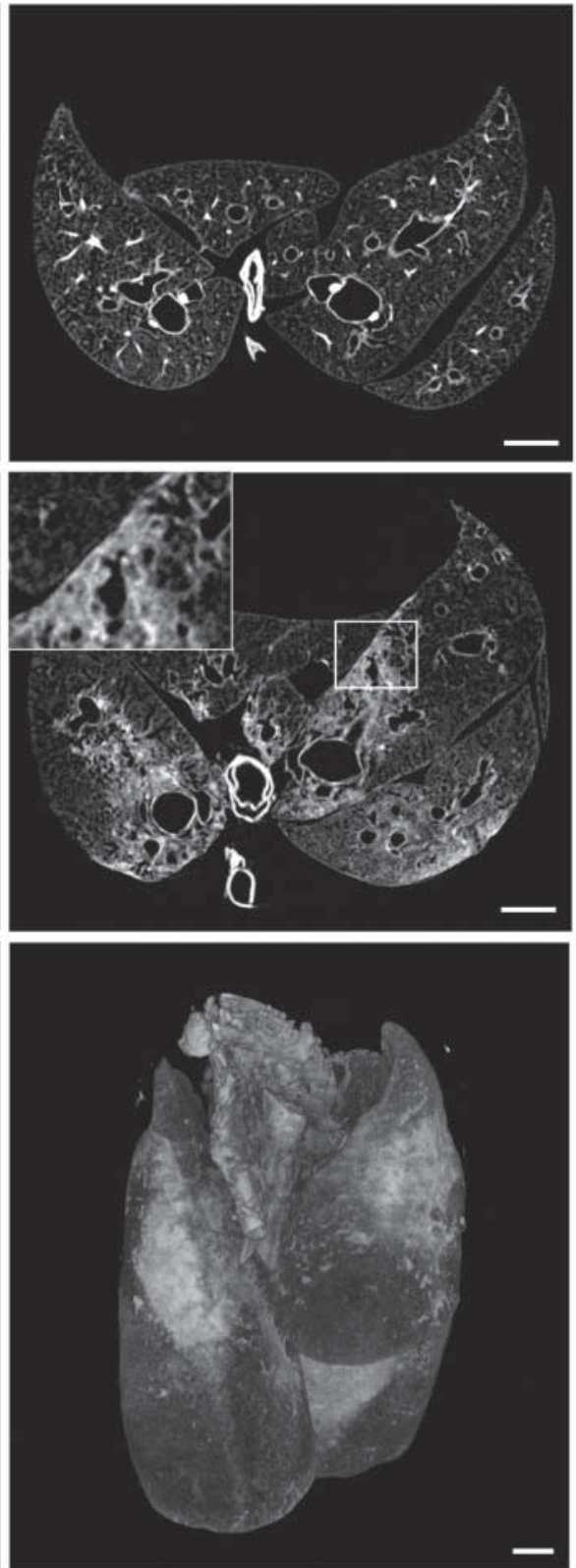

Day 28
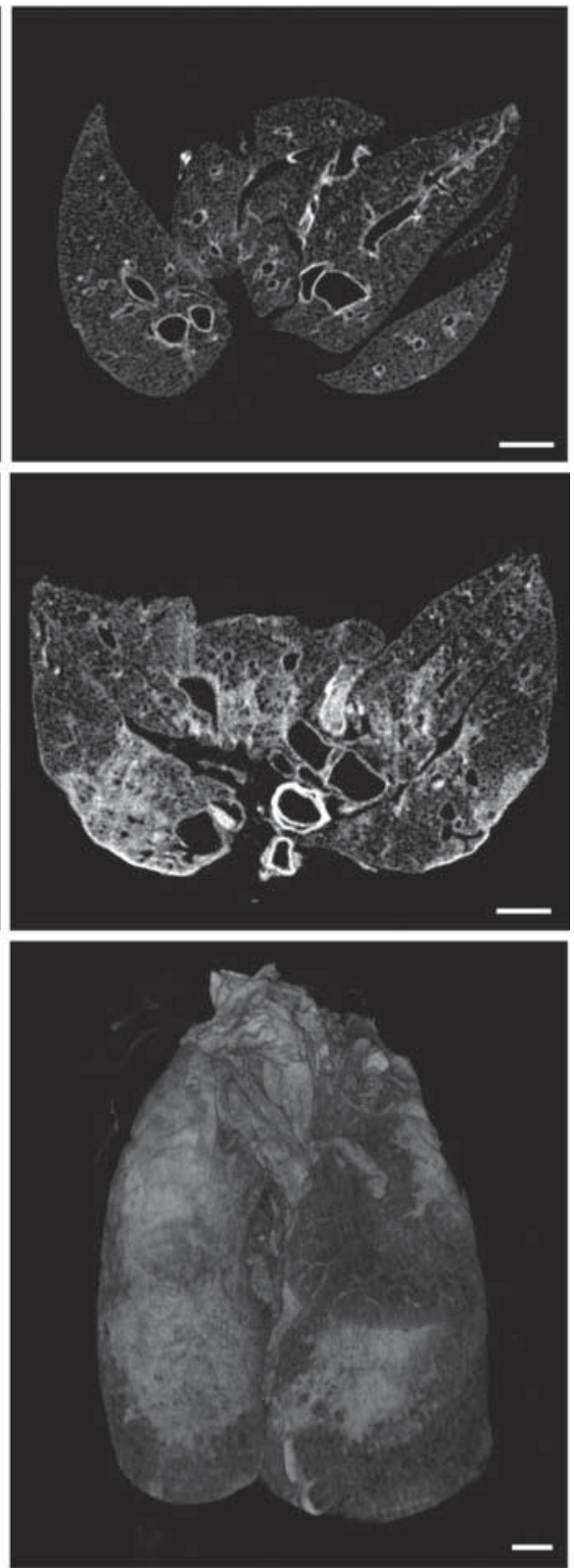

FIGURE 2 Ex vivo micro-computed tomography (micro-CT) lung images following bleomycin instillation. a) Representative mid-lung transaxial micro-CT sections from day 14 to day 28 post-saline/bleomycin instillation. Fibrotic regions comprising dense consolidation, traction bronchiectasis and subpleural scarring are highly evident in every lobe of lungs injured by bleomycin instillation. By day 28, the lung outline becomes distorted, which is particularly apparent on the dorsal (lower) surface. For orientation, the oesophagus (oe) and main bronchi (br) are labelled on the day 14 saline section. Day 21 post-bleomycin has an inset showing an enlargement $(\times 3)$ of the area bounded by the box, to highlight a region with traction bronchiectasis and dense consolidation, extending to the periphery of the lobe and adjacent to another lobe showing relatively normal lung tissue. b) $3 \mathrm{D}$ volume reconstructions of bleomycin-injured lungs from day 14 to day 28 , highlighting the reproducible dorsocentric lesion distribution. Scale bars $=1 \mathrm{~mm}$.

fibrotic change (reminiscent of fibrotic organising pneumonia) was still obvious by micro-CT, with thickening of interlobular septae and a lace-like pattern of fibrosis, enlarged airspaces, thickened subpleura and a massively distorted lung outline, indicating failure to fully resolve the BILF or completely restore the normal lung architecture (fig. 5b and c, fig. 6 and online supplementary videos 5 and 6). Micro-CT, therefore, revealed gross lung abnormalities which persisted for an extensive period following a single instillation of bleomycin. Histological examination demonstrated the presence of hyperplastic epithelium and/or bronchiolisation, and highly disturbed alveolar architecture. The volume $\times$ density readout by micro-CT was significantly higher than control at both 3 and 6 months post-bleomycin (fig. 5a). However, 

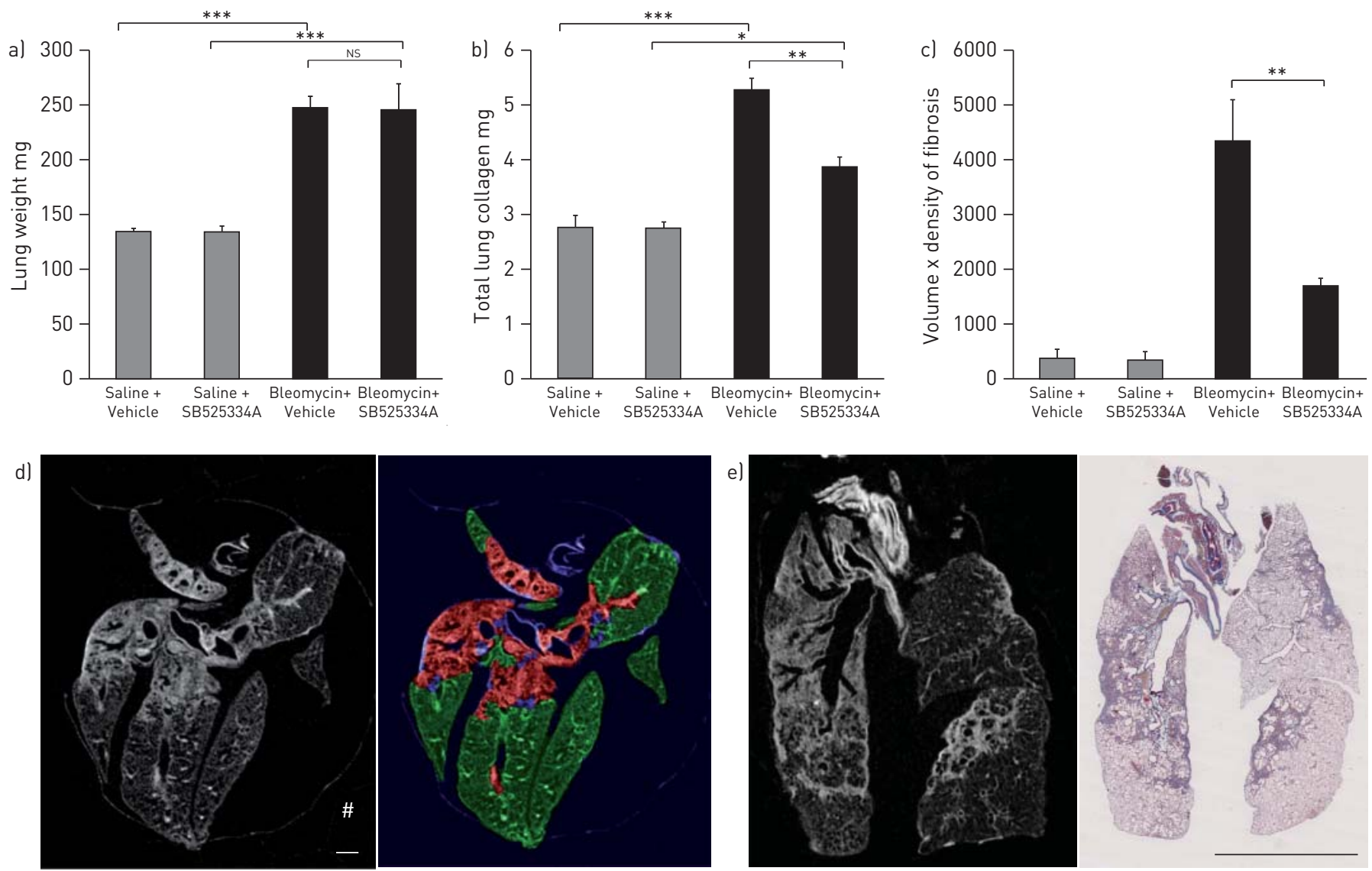

f)
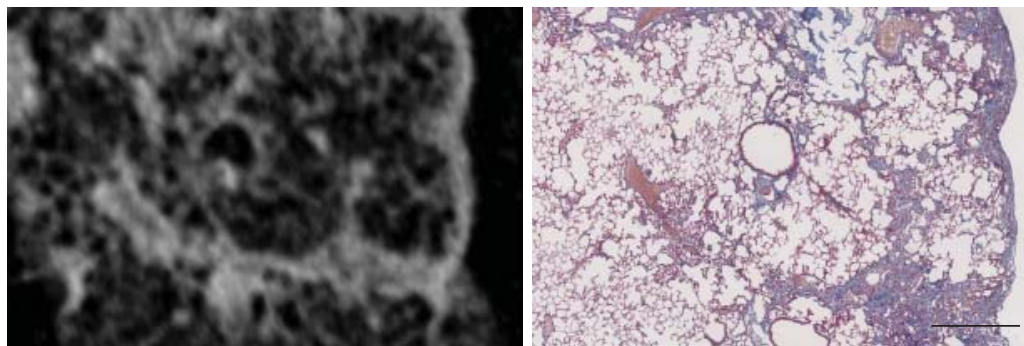

FIGURE 3 Therapeutic intervention during established fibrosis. a) Total lung weight at day 28 following bleomycin was significantly higher than in saline treated mice, but treatment with SB525334A by oral gavage twice daily from day 14 onwards did not attenuate the increase in lung weight. b) Total lung collagen accumulation (high-performance liquid chromatography determination of hydroxyproline) at day 28 was significantly abrogated by treatment with SB525334A. c) InForm (PerkinElmer, Cambridge, UK) software analysis of the micro-computed tomography (micro-CT) sections showed a significant increase in the fibrosis volume $\times$ density after bleomycin injury, which was also abrogated by SB525334A. d) Representative transaxial micro-CT section (left image) and the corresponding segmented image (right image) from the InForm software, showing pattern recognition of fibrosis (red), normal lung (green) and major airways/ vessels (blue); scale bar $=1 \mathrm{~mm}$. e) Fibrotic change on micro-CT (left image; coronal section) correlated perfectly with the collagen deposition (blue colour) seen in the corresponding Martius Scarlet Blue-stained histological section (right image); scale bar=5 mm. f) Higher power view of a fibrotic area from e); scale bar $=0.5 \mathrm{~mm}$. Data are presented as mean \pm SEM. $\mathrm{n}=6-8$ mice for hydroxyproline, $\mathrm{n}=3-4$ mice for micro-CT. NS: not significant. ${ }^{\star}: \mathrm{p}<0.05 ;{ }^{*}: \mathrm{p}<0.01$; ${ }^{* * *}: \mathrm{p}<0.001$ (ANOVA); ${ }^{*}$ CT signal from packaging material.

there was a significant diminution in this parameter at 6 months compared with 3 months, suggesting partial remodelling of the fibrotic change.

\section{Discussion}

To the best of our knowledge, this is the most thorough characterisation and benchmarking of the bleomycin model of lung fibrosis performed to date, using a cutting-edge micro-CT scanning and analytical approach to define a novel and sensitive end-point for preclinical drug evaluation. Identification of new therapies for the treatment of a disease such as IPF is very much dependent on the ability of experimental models to predict the eventual clinical outcome. In many studies (including our own previous work), prophylactic dosing schedules and/or the use of nonconditional genetically deficient mice have suggested 

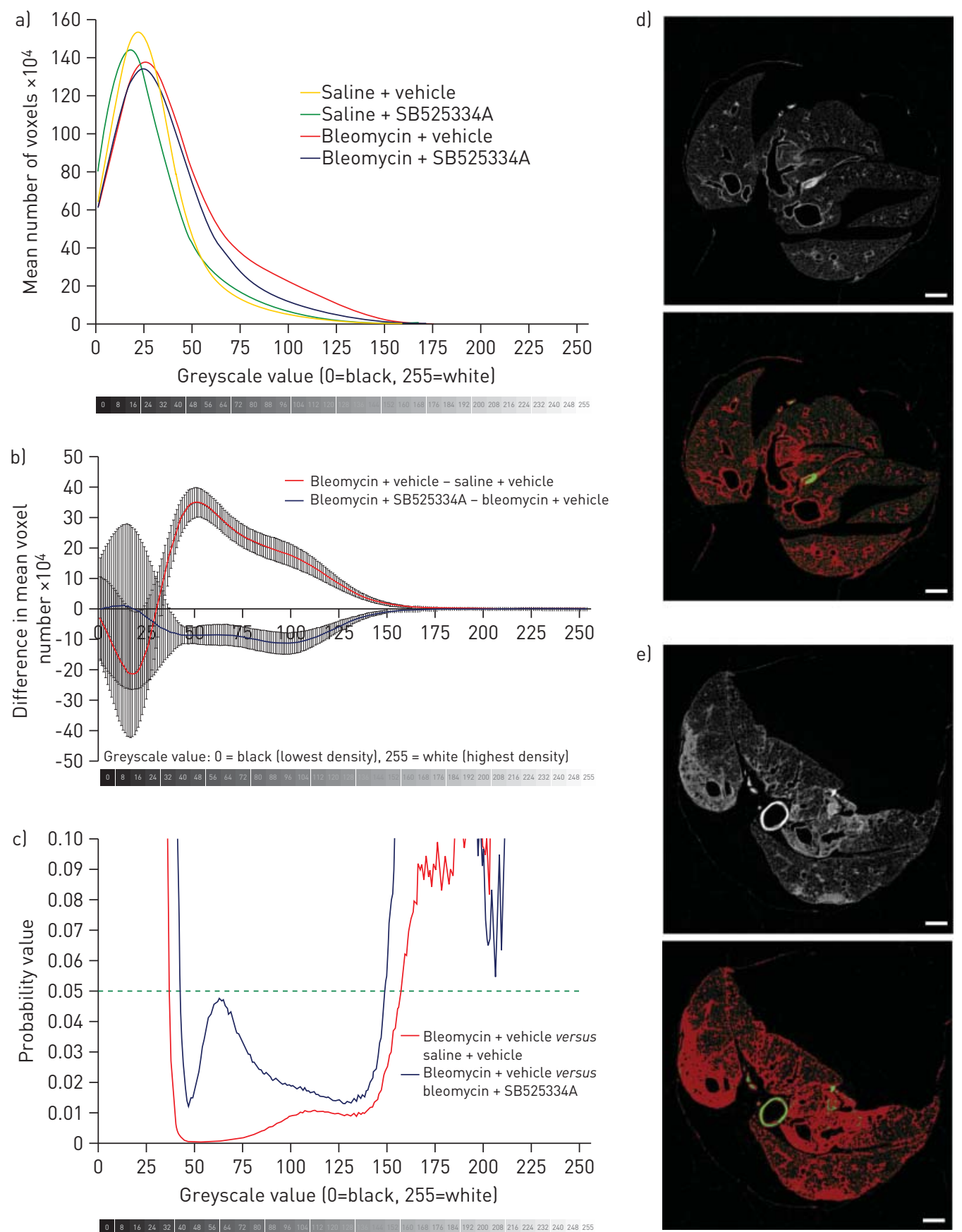

FIGURE 4 Voxel density analysis of micro-computed tomography (micro-CT) data. a) Histograms were plotted of voxel numbers at each greyscale density value (1-255); for clarity, data for a greyscale value of 0 (i.e. air) are excluded. The bleomycin and saline groups deviate at higher greyscale values, with a greater number of dense voxels due to bleomycin injury (indicative of dense fibrosis). b) The difference in number of voxels between the bleomycin + vehicle and saline + vehicle groups is clearly apparent; the bleomycin + SB525334A group has fewer voxels in this density range in comparison with the bleomycin + vehicle group (corrected for controls), which reaches c) statistical significance at greyscale values 42-137 ( $\mathrm{t}$-test). This greyscale range (red pixels) represents $\mathrm{d}$ ) airway walls in a saline + vehicle micro-CT slice, and e) dense fibrosis and airway walls in a bleomycin + vehicle micro-CT slice. Scale bars $=1 \mathrm{~mm}$.

the antifibrotic potential of a vast array of drug targets and key fibrotic pathways [3], yet the overwhelming majority of clinical trials have failed to reproduce the hoped-for translation of therapeutic benefit from mouse to male.

The limited number of studies where therapeutic dosing strategies have been employed arguably provide more clinically relevant data. Pirfenidone, for example, shows efficacy when dosed therapeutically during 

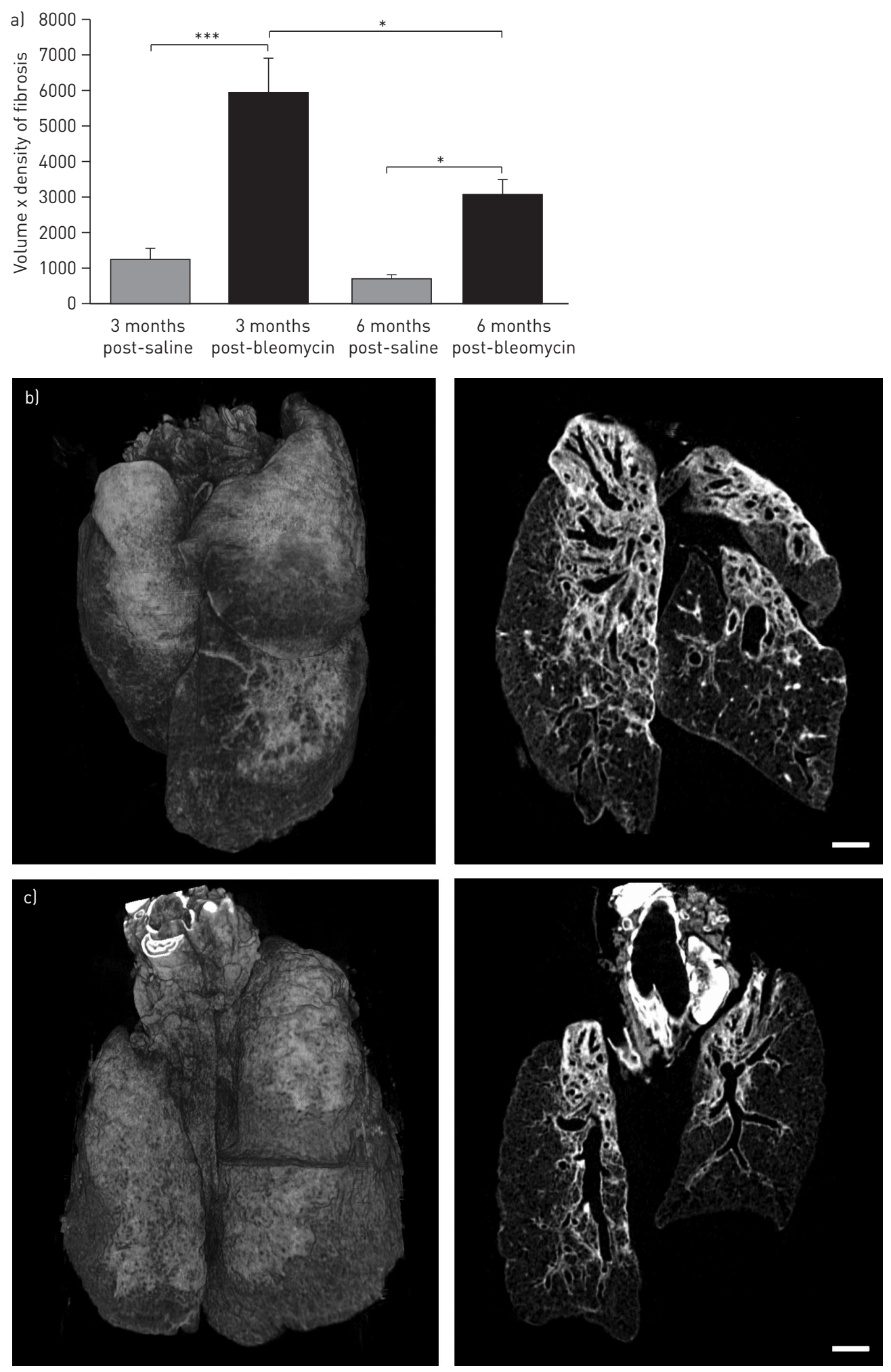

FIGURE 5 InForm (PerkinElmer, Cambridge, UK) image analysis of micro-computed tomography (micro-CT) data at longer timepoints following bleomycin injury. a) The same InForm image analysis was applied to micro-CT scans 3 and 6 months post-bleomycin. Significantly increased volume $\times$ density of fibrosis were observed, equivalent to data at days 21 and 28 (fig. $3 \mathrm{~b}$ and data not shown), showing that fibrotic lesions persist for $\geqslant 6$ months following a single instillation of bleomycin in this model. b, c) Representative 3D volume reconstructions and dorsal coronal CT sections from lungs 3 months and 6 months post-bleomycin, respectively, showing evidence for persistent fibrotic lesions, with a lace-like appearance reminiscent of fibrotic organising pneumonia. Scale bar $=1 \mathrm{~mm} . \mathrm{n}=4$ mice per group. 

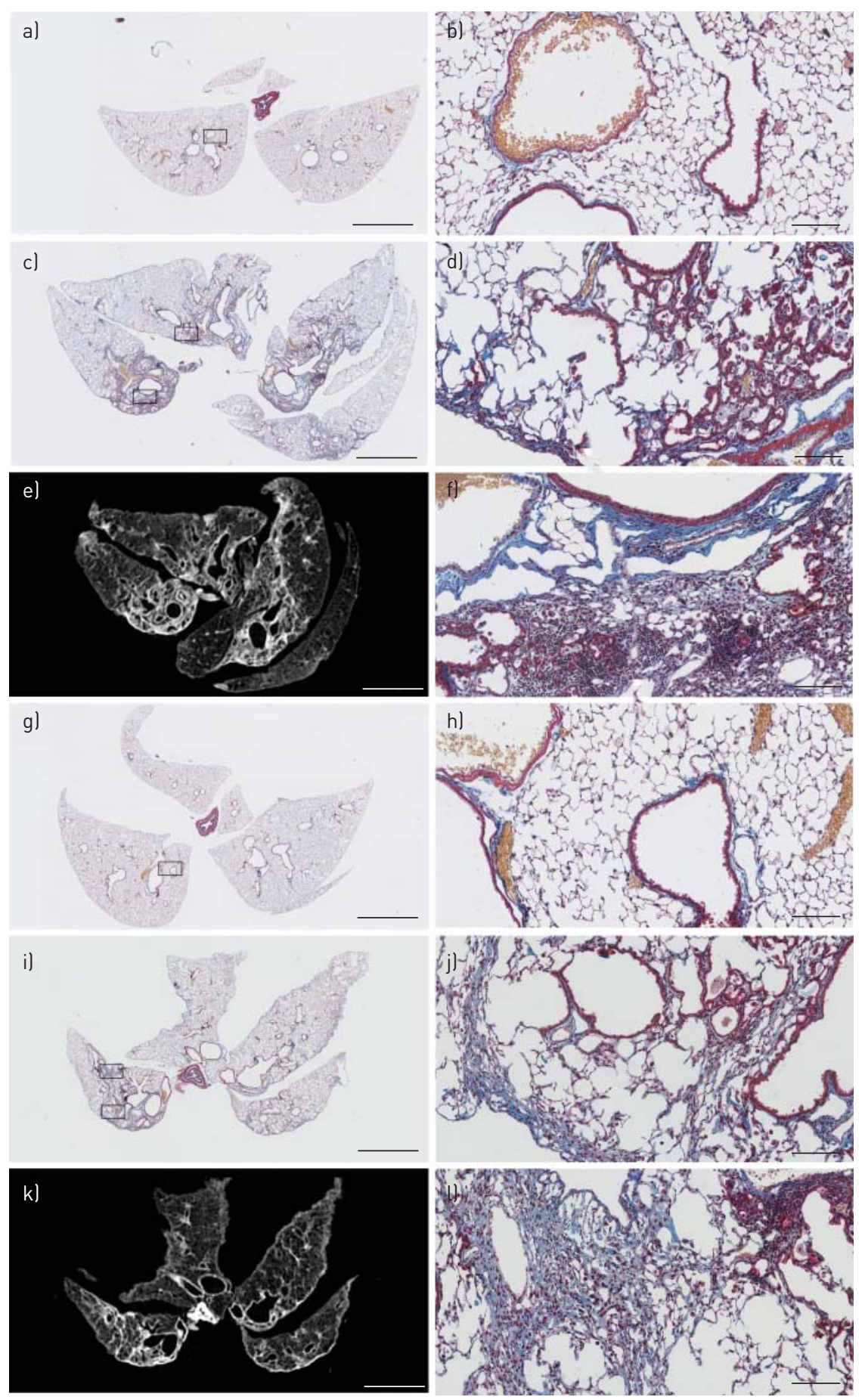

FIGURE 6 Histological appearance of lungs 3 and 6 months post-saline/bleomycin. a, b) Martius Scarlet Blue (MSB)stained section of a control lung 3 months post-saline reveals normal lung architecture at a) low and b) high power. c, d, e, f) 3 months post-bleomycin, gross abnormality of the lung architecture is still apparent, with c) a highly distorted lung outline apparent at low power; at higher power, there is clear evidence of d) hyperplastic epithelium/bronchiolisation, dense bands of mature scar tissue surrounding the f) bronchovascular bundles and f) frequent lymphocytic infiltrates; e) the equivalent mid-upper lung transaxial micro-computed tomography (micro-CT) section is also shown. g, h) MSBstained section of a control lung 6 months post-saline reveals normal lung architecture at g) low and h) high power. i, i, k, 1) By 6 months post-bleomycin, fibrotic lesions were still evident and the lung outline remained severely distorted as shown at i) low power; hyperplastic epithelium was particularly evident in areas of milder fibrosis, as shown in j), suggesting an association with repair of the dense lesions; 1) overall cellularity appears diminished, but dense bands of scar tissue remain; $\mathrm{k}$ ) the equivalent mid-upper lung transaxial micro-CT section is also shown. All sections were stained with MSB. For low-power sections, scale bar $=2 \mathrm{~mm}$; high-power sections, scale bar $=100 \mu \mathrm{m}$ (boxes on low power sections show the location of magnified areas). 
bleomycin-induced lung fibrosis in hamsters and mice $[6,13]$, and has received marketing authorisation for the treatment of mild-to-moderate IPF in Europe. In contrast, oral prednisolone given therapeutically to rats following bleomycin administration does not ameliorate fibrosis $[9,14]$. A recent clinical trial of the tyrosine kinase inhibitor, imatinib, failed to demonstrate any beneficial effects on survival or lung function in IPF [15]; therapeutic dosing of imatinib in mice following bleomycin exposure also failed to show efficacy [8]. It could therefore be argued that the experimental model of lung fibrosis induced by bleomycin may have the potential to be predictive of clinical outcome, if appropriate dosing strategies and end-points are employed.

In this study we have carefully defined a window of opportunity between 14 and 28 days after a single oropharyngeal instillation of bleomycin ( $50 \mathrm{IU}$ per mouse in $50 \mu \mathrm{L}$ saline), where there is progression of the established fibrotic lesions, as defined by a further increase in the amount of total lung collagen and a maturation of the deposited collagen fibres on histology. Although this represents a rapid and acute worsening of the fibrosis, the underlying pathomechanisms are probably relevant in terms of human disease.

Small-animal imaging, using micro-CT, volumetric flat panel CT [16] or magnetic resonance imaging [17], for example, is gaining in popularity for investigating morphological changes in the murine lung. Since these are minimally invasive and nondestructive approaches, they can also be applied to longitudinally investigate the development of lung fibrosis in mice and rats in vivo; however, the degree of resolution is constrained by respiratory and cardiac motion [18] and, for CT, the dose of radiation that can safely be administered. Our ex vivo micro-CT scanning approach clearly identified the fibrotic lesions from 14 days onwards, with a highly reproducible mid- to upper-lung and dorsocentric distribution pattern, and negated the practical and analytical difficulties associated with imaging live animals, thereby opening up the adoption of this methodology by other laboratories. Moreover, by analysing the whole lung we avoided the sampling error/bias associated with histological analyses [19].

Elegant studies have compared and contrasted in vivo and ex vivo micro-CT scanning of normal murine lungs [11] or highlighted differences in lung structure between mouse strains [20]. Few studies, however, have addressed the use of micro-CT for evaluation of the degree of fibrotic change in murine models of lung fibrosis. Previous studies [21-23] used in vivo micro-CT to investigate fibrotic changes in the adenoviral TGF- $\beta 1$ overexpression-induced or bleomycin models of lung fibrosis in rats or mice with associated voxel sizes of $\sim 155 \mu \mathrm{m}, 94 \mu \mathrm{m}$ or $35 \mu \mathrm{m}$. These studies used different approaches to segment the tissue into either fibrotic [21] or aerated lung volumes [22], or used semiquantitative pathological scoring [23], but the utility of these approaches has not yet been validated with a drug intervention strategy. A recent study by JIN et al. [24] used micro-CT (with $\sim 44.25-\mu \mathrm{m}$ spatial resolution) to investigate the therapeutic effects of rosiglitazone during BILF in mice; they reported beneficial changes in a qualitative radiological assessment, but did not provide a fully quantitative measure of fibrotic change. It is worth mentioning that the study by AsK et al. [21] also investigated lung function (pressure-volume loops on mechanical ventilation) and demonstrated a strong correlation between lung stiffness and the degree of fibrosis on micro-CT, highlighting that lung function parameters are also a highly relevant end-point measure in fibrosis models.

Our micro-CT study has been benchmarked using a carefully conducted therapeutic dosing strategy with a TGF- $\beta$ R-1 kinase (ALK5) inhibitor. ALK5 inhibition was selected as an appropriate proof of concept, given the undisputed importance of the TGF- $\beta$ signalling pathway as a major driver of the pro-fibrotic response; ALK5 inhibition with the small molecule inhibitor SD-208 was shown to be of therapeutic benefit in the AdTGF- $\beta 1$-induced model of lung fibrosis [25], while prophylactic dosing with SB525334A attenuated various histopathological alterations of the fibrotic lung during BILF [26]. In line with the SD-208 data [25], our therapeutic dosing strategy with SB525334A also significantly attenuated fibrotic progression. Dosing commenced when the lungs would already have had a $\sim 60 \%$ increase in total lung collagen (i.e. at 14 days post-bleomycin); ALK5 inhibition completely blocked the expected progression, validating the major pro-fibrotic role of TGF- $\beta$ signalling. HPLC determination of total lung collagen and micro-CT evaluation of fibrosis were in close agreement in terms of the efficacy of SB525334A, with a roughly 55-60\% attenuation of the fibrotic response. Importantly, however, micro-CT gave a larger window for detecting a therapeutic effect. Significantly, this response was determined with as few as three or four mice per group, in contrast to the 8-11 mice normally used for HPLC determination, thus reducing the number of experimental animals required; moreover, the same lungs used for micro-CT could then be processed for histological examination, further refining and reducing the required number of mice. The approach is necessarily dependent on a highly standardised and homogeneous lung insufflation/fixation protocol to avoid misclassification of tissue artefacts; however, in a separate study, HPLC analysis of lung hydroxyproline was also successfully performed on lungs immediately after micro-CT analysis (rather than histology), and revealed a significant correlation between total lung collagen and micro-CT evaluation $\left(\mathrm{r}^{2}=0.7553, \mathrm{p}<0.001\right)$. In terms of radiological assessment, micro-CT clearly highlighted areas of dense 
consolidation, traction bronchiectasis, interlobular septal thickening and subpleural scarring, and these areas corresponded perfectly to areas of fibrosis identified histologically. This work lends further support to the validity and improved sensitivity of micro-CT as a true outcome measure of fibrotic severity in BILF.

Of interest, we also assessed the commonly used Sircol assay during the temporal response to bleomycin. The proportion of lung collagen detected by Sircol accounted for only $9 \%$ of the total lung collagen determined by HPLC analysis. No correlation between the two measures was determinable. Moreover, Sircol revealed an increase in the collagen signal at 7 days post-bleomycin, which was not reproduced by HPLC determination. In a recent paper by LAREU et al. [27], a detailed analysis of the Sircol assay highlighted a problem due to interference of noncollagenous proteins, such as serum proteins, unless the protocol is appropriately modified; we would speculate that the increased Sircol signal at day 7 may therefore reflect increased vascular leak at this timepoint [10], and would question the validity of the Sircol assay as a standalone end-point measure of fibrosis, unless appropriately modified.

A major criticism of BILF as a model of IPF is the widespread acceptance that the fibrotic lesions resolve rapidly, often within 5-6 weeks following bleomycin administration [28, 29], which is clearly at odds with the human condition which is chronic and progressive. Our data instead showed that while the fibrotic lesions do not continue to progress, they do persist for $\geqslant 6$ months following a single oropharyngeal instillation of bleomycin. Indeed, at 3 months post-bleomycin, the increase in total lung collagen was equivalent to that seen at 28 days, implying little or no resolution of the fibrotic response in this timeframe. By 6 months post-bleomycin, micro-CT continued to reveal the presence of a highly altered lung architecture, reminiscent of fibrotic organising pneumonia, and associated with fibrosis around bronchovascular bundles, subpleural scarring and a massively distorted lung outline. Histologically, the overall cellularity of the fibrotic lesions appeared decreased (in terms of inflammatory infiltrate and fibroblasts), and they were instead composed of dense bands of scar tissue, associated with abundant hyperplastic epithelium and/or bronchiolisation, which are histological hallmarks of IPF. Previously, hyperplastic epithelium has only been consistently observed in a repetitive bleomycin dosing model [30]. Our data suggest that these features are associated with remodelling of the fibrosis, but there does not appear to be a marked restoration of the alveolar architecture at this late timepoint. Under these conditions therefore, we do not observe complete resolution of the fibrotic response to bleomycin, which opens up the possibility for assessing therapeutic approaches capable of initiating lung repair, rather than just halting fibrosis. Our data also reiterate the temporal heterogeneity of the fibrotic response instigated by the various permutations of the bleomycin model [5].

In conclusion, this work demonstrates that the experimental model of lung fibrosis induced by oropharyngeal bleomycin administration can be a highly useful model for establishing the therapeutic efficacy of target compounds to block fibrotic progression of lesions which do not rapidly resolve. It also highlights the importance of a thorough characterisation of each laboratory's permutation of the bleomycin model, to define the most appropriate dosing strategies and to understand the natural history of the fibrotic response and associated fibrogenic mechanisms.

\section{Acknowledgements}

We would especially like to thank Penny Shaw (University College London Hospital, London, UK), for expert evaluation and interpretation of the micro-CT data and Alejandro Ortiz-Stern (University College London, London, UK) for highly useful discussions regarding resolution of fibrosis. We would also like to thank Steve E. Bottoms (University College London) and Mike Haase (GlaxoSmithKline, Stevenage, UK) for expert technical assistance with histology and micro-CT, respectively.

\section{References}

1 Coultas DB, Zumwalt RE, Black WC, et al. The epidemiology of interstitial lung diseases. Am J Respir Crit Care Med 1994; 150: 967-972.

2 Datta A, Scotton CJ, Chambers RC. Novel therapeutic approaches for pulmonary fibrosis. Br J Pharmacol 2011; 163: 141-172.

3 Moeller A, Ask K, Warburton D, et al. The bleomycin animal model: a useful tool to investigate treatment options for idiopathic pulmonary fibrosis? Int J Biochem Cell Biol 2008; 40: 362-382.

4 Moore BB, Hogaboam CM. Murine models of pulmonary fibrosis. Am J Physiol Lung Cell Mol Physiol 2008; 294: L152-L160.

5 Scotton CJ, Chambers RC. Bleomycin revisited: towards a more representative model of IPF? Am J Physiol Lung Cell Mol Physiol 2010; 299: L439-L441.

6 Kakugawa T, Mukae H, Hayashi T, et al. Pirfenidone attenuates expression of HSP47 in murine bleomycin-induced pulmonary fibrosis. Eur Respir J 2004; 24: 57-65.

7 Chaudhary NI, Roth GJ, Hilberg F, et al. Inhibition of PDGF, VEGF and FGF signalling attenuates fibrosis. Eur Respir J 2007; 29: 976-985.

8 Aono $\mathrm{Y}$, Nishioka $\mathrm{Y}$, Inayama $\mathrm{M}$, et al. Imatinib as a novel antifibrotic agent in bleomycin-induced pulmonary fibrosis in mice. Am J Respir Crit Care Med 2005; 171: 1279-1285. 

bleomycin model. Am J Respir Crit Care Med 2006; 173: 769-776.

10 Scotton CJ, Krupiczojc MA, Königshoff M, et al. Increased local expression of coagulation factor X contributes to the fibrotic response in human and murine lung injury. J Clin Invest 2009; 119: 2550-2563.

11 Vasilescu DM, Knudsen L, Ochs M, et al. Optimized murine lung preparation for detailed structural evaluation via micro-computed tomography. J Appl Physiol 2012; 112: 159-166.

12 Grygielko ET, Martin WM, Tweed C, et al. Inhibition of gene markers of fibrosis with a novel inhibitor of transforming growth factor-beta type I receptor kinase in puromycin-induced nephritis. J Pharmacol Exp Ther 2005; 313: 943-951.

13 Iyer SN, Margolin SB, Hyde DM, et al. Lung fibrosis is ameliorated by pirfenidone fed in diet after the second dose in a three-dose bleomycin-hamster model. Exp Lung Res 1998; 24: 119-132.

14 McGrath EE, Millar AB. Hot off the breath: triple therapy for idiopathic pulmonary fibrosis - hear the PANTHER roar. Thorax 2012; 67: 97-98.

15 Daniels CE, Lasky JA, Limper AH, et al. Imatinib treatment for idiopathic pulmonary fibrosis: randomized placebocontrolled trial results. Am J Respir Crit Care Med 2010; 181: 604-610.

16 Wielpütz MO, Eichinger M, Zhou Z, et al. In vivo monitoring of cystic fibrosis-like lung disease in mice by volumetric computed tomography. Eur Respir J 2011; 38: 1060-1070.

17 Babin AL, Cannet C, Gérard C, et al. Noninvasive assessment of bleomycin-induced lung injury and the effects of short-term glucocorticosteroid treatment in rats using MRI. J Magn Reson Imaging 2011; 33: 603-614.

18 Bartling SH, Kuntz J, Semmler W. Gating in small-animal cardio-thoracic CT. Methods 2010; 50: 42-49.

19 Hsia CC, Hyde DM, Ochs M, et al. An official research policy statement of the American Thoracic Society/ European Respiratory Society: standards for quantitative assessment of lung structure. Am J Respir Crit Care Med 2010; 181: 394-418.

20 Thiesse J, Namati E, Sieren JC, et al. Lung structure phenotype variation in inbred mouse strains revealed through in vivo micro-CT imaging. J Appl Physiol 2010; 109: 1960-1968.

21 Ask K, Labiris R, Farkas L, et al. Comparison between conventional and "clinical" assessment of experimental lung fibrosis. J Transl Med 2008; 6: 16.

22 Rodt T, von Falck C, Dettmer S, et al. Micro-computed tomography of pulmonary fibrosis in mice induced by adenoviral gene transfer of biologically active transforming growth factor- $\beta 1$. Respir Res 2010; $11: 181$.

23 Lee HJ, Goo JM, Kim NR, et al. Semiquantitative measurement of murine bleomycin-induced lung fibrosis in in vivo and postmortem conditions using microcomputed tomography: correlation with pathologic scores - initial results. Invest Radiol 2008; 43: 453-460.

24 Jin GY, Bok SM, Han YM, et al. Effectiveness of rosiglitazone on bleomycin-induced lung fibrosis: assessed by micro-computed tomography and pathologic scores. Eur J Radiol 2012; 81: 1901-1906.

25 Bonniaud P, Margetts PJ, Kolb M, et al. Progressive transforming growth factor $\beta 1$-induced lung fibrosis is blocked by an orally active ALK5 kinase inhibitor. Am J Respir Crit Care Med 2005; 171: 889-898.

26 Higashiyama H, Yoshimoto D, Kaise T, et al. Inhibition of activin receptor-like kinase 5 attenuates bleomycininduced pulmonary fibrosis. Exp Mol Pathol 2007; 83: 39-46.

27 Lareu RR, Zeugolis DI, Abu-Rub M, et al. Essential modification of the Sircol Collagen Assay for the accurate quantification of collagen content in complex protein solutions. Acta Biomater 2010; 6: 3146-3151.

28 Chung MP, Monick MM, Hamzeh NY, et al. Role of repeated lung injury and genetic background in bleomycininduced fibrosis. Am J Respir Cell Mol Biol 2003; 29: 375-380.

29 Lawson WE, Polosukhin VV, Stathopoulos GT, et al. Increased and prolonged pulmonary fibrosis in surfactant protein C-deficient mice following intratracheal bleomycin. Am J Pathol 2005; 167: 1267-1277.

30 Degryse AL, Tanjore H, Xu XC, et al. Repetitive intratracheal bleomycin models several features of idiopathic pulmonary fibrosis. Am J Physiol Lung Cell Mol Physiol 2010; 299: L442-L452. 